Acta Biotheretica 37: 315-319, 1988.

(C) 1988 Kluwer Academic Publishers. Printed in the Netherlands.

IDEAS IN THEORETICAL BIOLOGY

EVOLUTIONARY AND CLASSICAL CONCEPTS OF HOMOLOGY: A REPLY TO ABOITIZ

\title{
MARK WILKINSON
}

Aboitiz (1988) proposed a return to pre-Darwinian concepts of homology. The main points raised by this author can be summarized as follows.

1. A distinction can be drawn between the modern evolutionary concept of homology, indicating a common evolutionary origin of morphological traits, genes or proteins and preDarwinian concepts of homology (here termed 'classical homology') indicating a common embryonic origin or set of topographical relations shared by morphological traits.

2. Criteria of classical homology are empirically testable.

3. Classical homologies are very useful for the "elucidation of taxonomies".

4. The evolutionary concept of homology is not a criterion for classification. This kind of homology is established after taxonomies are "elucidated". The evolutionary concept is thus "not very practical in phylogenetic classification".

5. There is no expression representing the concept of 'classical homology' because two structures conforming to the classical criteria would not constitute homologies in the evolutionary sense if they arose independently.

6. The classical homology concept is not applicable to molecular biology. 
7. There is a renaissance in the study of morphogenesis in which evolutionary considerations are secondary to studies of ontogenetic processes.

8. The classical homology concept, necessarily restricted to morphology, is more useful under the new morphogenetic perspective and hence preferable.

I wish to take issue with several of these points.

Assessment of classical homology is empirically testable inasmuch as criteria can be applied on a set of classical homologies established. However, it is not certain what this concept delimits except a set of traits with a certain correspondence. The correspondence may be real, but it is not clear why such correspondences should be preferred over alternative types of correspondence (the pre-Darwinian concept of analogy for instance) for use in the construction of classifications. That classical homologies are useful for the construction of classifications cannot be doubted because many pre-Darwinian classifications were based upon them, but other correspondences can and have been usefully employed as a basis for classification (e.g. estimates of overall similarity). It is important to draw a distinction between classifications and the relations upon which they are based. Classifications are not "elucidated", rather relations or correspondences are elucidated (or simply perceived) and classifications are constructed based on a subset of all of the many kinds of relations that can be elucidated.

Far from not being practical for phylogenetic classification, the concept of evolutionary homology is extremely important. Phylogenetic classifications are based on phylogenetic inferences. In attempting to elucidate phylogenetic relations use is made of traits that show certain correspondences including morphological traits which meet the criteria of classical homology, and molecular traits such as DNA sequences and the electrophoretic mobilities of proteins. These are taken as putative evolutionary homologues, which would explain their correspondence, and thus as evidence of common ancestry of the taxa that share them. 
Phylogenetic inference is complicated by the fact that usually not all the 'putative homologues' are evolutionary homologues. This is the case when some putative homologues provide evidence for mutually incompatible phylogenetic relationships. Methods of phylogenetic inference are intended to weigh contradictory evidence and determine the 'best' phylogenetic hypothesis relative to this evidence. Preferred phylogenetic hypotheses can be used as a basis for the construction of phylogenetic classifications, but it is not the case that evolutionary homology is established after the classification has been constructed. Preferred phylogenetic hypotheses are based on preferred hypotheses of evolutionary homology as revealed by application of the methods of phylogenetic analysis. Thus for any phylogenetic classification (based on a preferred phylogenetic hypothesis) evolutionary homology is clearly a criterion of the classification because it is the assessment of evolutionary homology that provides the evidential support upon which the phylogenetic hypothesis is based.

Given that incompatibilities occur in groups delimited by different traits all of which pass the criteria of classical homology, this concept appears to be no more useful as a basis for the construction of classifications than the evolutionary concept. Indeed, since the concept of classical homology can be used only with arbitrary or subjective decisions about which of the incompatible classical homologues are to be used for the purposes of classification and which are to be discounted, its use is less satisfying than the use of the evolutionary concept of homology and the associated methods for distinguishing homology from homoplasy. These methods may be far from perfect but the reason for their application is clear and not arbitrary.

In the context of phylogenetic studies, putative homologues (including classical homologies of the morphological type) are interpreted as either evolutionary homologues or homoplasies (the latter term subsuming parallelisms, reversals and convergences). thus although no single term exists to describe 
classical homology and classical homology can be meaningfully described by one of these terms. Alternatively, the precise kind of classical homology could simply be referred to by the more informative terms 'embryological correspondence' and 'topographical correspondence' which can be employed without any evolutionary implication.

Given that the concept of classical homology is not applicable to molecular biology (although proteins have ontogenies and genes may exhibit topographical relations) this would be one reason to prefer the more general evolutionary homology concept which can indicate the same kind of relation that can exist between attributes as diverse as morphological structures and genes.

Any renaissance in the study of morphogenesis is most welcome. The investigation of morphogenetic processes can and often does proceed independently of evolutionary considerations which may thus be secondary (i.e. not the prime focus of the research programme) to questions of developmental mechanics. However, a more complete understanding of morphogenesis would stem from the answering of questions concerning the origins of morphogenetic processes in combination with a knowledge of the processes themselves. Both kinds of study are worthy and complementary. It is not necessary and it is potentially counterproductive to attempt to establish the primacy of mechanistic studies over evolutionary ones or vice versa. Furthermore, it is by no means clear how a return to the classical concept of homology would be more useful for the new perspective associated with the renaissance of studies of morphogenetic processes and how the concepts of evolutionary homology and homoplasy would be any less useful. With the proposed restriction of homology to morphological traits and return to the classical concept, it would make no sense to describe the shared genetic basis of evolutionarily homologous morphological traits as homologous themselves and there would simply be no term to describe evolutionary homology. Aboitiz's (1988) proposal for capture of the term homology by a nonevolutionary discipline would not benefit that discipline and 
would deprive evolutionary studies of morphogenesis, morphology and molecular biology of a widely accepted term for a central concept. I suggest therefore that the proposal should be rejected.

\section{REFERENCES}

Aboitiz, F. (1988). Homology: a comparative or a historical concept? Acta Biotheoretica 37: 27-29.

$$
\begin{array}{r}
\text { Mark Wilkinson } \\
\text { Museum of Zoology } \\
\text { and Department of Biology } \\
\text { University of Michigan } \\
\text { Ann Arbor, MI } 48109 \\
\text { USA }
\end{array}
$$

